\title{
DIE FORSCHUNGSSTELLE FÜR VÖLKERRECHT UND AUSLÄNDISCHES ÖFFENTLICHES RECHT DER UNIVERSITÄT HAMBURG
}

\begin{abstract}
Die Forschungsstelle für Völkerrecht und ausländisches öffentliches Recht der Universität Hamburg wurde im Jahre 1946 durch Erlaß des Senats der Freien und Hansestadt Hamburg gegründet. Sinn der Gründung war es, zwei ältere Hamburger Forschungsinstitute, die infolge der Kriegsereignisse im wesentlichen nur noch als Bibliotheken vorhanden waren, organisatorisch zusammenzufassen und für eine zeitgemäße Erforschung des Völkerrechts, des ausländischen öffentlichen Rechts und der Auswärtigen Politik wieder arbeitsfähig zu machen. Die ersten Direktoren des neuen Instituts waren Professor Rudolf Laun und Professor Friedrich Schack. Dem Institut standen zunächst drei Referenten und zwei Assistenten zur Verfügung sowie die Bibliotheken der alten Institute mit anfänglich insgesamt ca. 35000 Bänden juristischer und sozialwissenschaftlicher Literatur und ein umfangreiches Zeitungsarchiv. Das neue Institut erhielt in den ersten Jahren insbesondere die Aufgabe, gutachtlich zu besatzungsrechtlichen Fragen und zur Staatsangehörigkeit vieler durch die Kriegsereignisse nach Deutschland gelangter Personen Stellung zu nehmen sowie Anwärter für den diplomatischen Dienst auszubilden.

Von Anfang an wurde die Auftragsforschung durch Grundlagenforschungen ergänzt. Die Grundlagenforschungen erfolgten zunächst im Bereich des allgemeinen Völkerrechts und des Staatsangehörigkeitsrechts und wurden seit dem Jahre 1948 in dem gemeinsam mit dem Institut für Internationales Recht an der Universität Kiel herausgegebenen "Jahrbuch für internationales und ausländisches öffentliches Recht"1 sowie in den Reihen "Abhandlungen“, „Sammlung geltender Staatsangehörigkeitsgesetze“ und "Hektographierte Veröffentlichungen“" interessierten Kreisen zugänglich gemacht. Die Tradition der Universität Hamburg, Wissenschaft mit dem Blick auf Übersee zu pflegen, hatte die von der Forschungsstelle übernommenen Institute und ihre Bibliotheken geprägt. So wird es verständlich, daß der Ausbau des neuen Instituts auch in dieser Richtung erfolgte. Zum Verständnis der Arbeitswcise und des Aufbaus des Instituts dürfte es daher dienlich sein, kurz die beiden übernommenen bzw. verwalteten Institute zu skizzieren.
\end{abstract}

\section{Das Institut für Kolonialrecht der Universität Hamburg}

Im Jahre 1908 war in Hamburg das Kolonialinstitut gegründet worden, zu dessen Aufgabe es im „Bericht über das erste Studienjahr“ heißt: „Überall ..., wohin der Europäer sich wendet, sind Kulturen vorhanden, ... Hier erschien es selbstverständlich, daß das Studium der Kultur vor der Ausreise ... ein richtiges Urteil und besseres Abwägen gestattet." Dieser Satz dürfte bis heute zu beherzigen sein. Unter den fünf ersten Lehrstühlen des Kolonialinstituts fand sich auch ein Lehrstuhl für öffentliches Recht, der Kolonialrecht und, wie es damals hieß, „Eingeborenenrecht" umfaßte. Der erste Inhaber dieses Lehrstuhls war Richard Thoma, der allerdings nach einem Semester Hamburg den Rücken kehrte. An seine Stelle trat Kurt Perels, ein Gelehrter, der sich besonders dem Seerecht und der afrikanischen Rechtsethnologie zuwandte. Er legte eine Sammlung afrikanischer Urkunden, Hoheitszeichen und Strafwerkzeuge an, die leider den Bombenangriffen des Zweiten Weltkrieges zum Opfer gefallen ist. Unter Perels wurde eine Reihe kolonialrechtlicher Untersuchungen durchgeführt und veröffentlicht, die Pflege der Erforschung des

1 Erscheint heute unter dem Titel Jahrbuch für internationales Recht ${ }^{\alpha}$ im Verlag Vandenhoeck \& Ruprecht, Göttingen. 
„Eingeborenenrechts“ wurde jedoch aus „praktischen Gründen“ zurückgestellt, weil es „auf der einen Seite ... noch zu wenig erforscht" war und „auf der anderen Seite, da jeder Stamm oder ein noch kleinerer Bezirk" sein eigenes Recht hatte, Bände gefüllt hätte ${ }^{2}$. Mag diese Begründung auch nicht unbedingt überzeugen, so bleibt doch festzustellen, daß die Auslandsrechtsforschung mit Blick auf Úbersee seit 1919 eingestellt wurde.

Im Jahre 1940 wurde der Versuch unternommen, das alte Seminar als Institut für Kolonialrecht wiederzubeleben. Das Institut sah seine wichtigste Aufgabe darin, neben der Erstellung von Bibliographien über das Kolonialrecht aller europäischen Staaten Untersuchungen über die Rechtsanschauungen der Afrikaner vorzubereiten. Es wurden Arbeiten über Sklavenhandel und Haussklaverei in Kamerun und Togo, über die bodenrechtlichen Vorstellungen im belgischen Kongo und über die Staatsorganisation im alten Ruanda begonnen, die allerdings nicht mehr zur Veröffentlichung gelangten. Am Ende des Zweiten Weltkriegs blieb von dem Institut lediglich eine Bibliothek von ca. 3000 Bänden völkerrechtlichen und kolonialrechtlichen Schrifttums übrig.

\section{Das Institut für Auswärtige Politik}

Das Institut für Auswärtige Politik verdankt sein Entstehen der katastrophalen Erschütterung, die der Erste Weltkrieg unter den beteiligten und nicht beteiligten Völkern ausgelöst hatte. Während der Friedenskonferenz in Paris im Jahre 1919 gelangten die Vertreter der beteiligten Nationen zu der Einsicht, daß die Gesetzmäßigkeiten in den Völkerbeziehungen zu erforschen seien und daß damit wichtige Grundlagen $\mathrm{zu}$ einem dauernden Frieden gefunden werden könnten. Ebenso wie in anderen Ländern (Royal Institute of International Affairs in England, Council on Foreign Relations und Foreign Policy Association in den USA) fanden sich auch in Deutschland Interessierte zusammen, um auf dem Gebiet der auswärtigen Politik zu arbeiten. Hamburg schien als Sitz eines Instituts für Auswärtige Politik besonders geeignet, da „von hier aus seit langem bewußte Kritik an der amtlichen Politik geübt worden und selbständige Einsicht in das Verhältnis Deutschlands zu anderen Völkern, besonders über See, gewonnen worden war" (MendelssohnBartholdy).

Im Jahre 1921 gründete Professor Albrecht Mendelssohn-Bartholdy, Professor für Auslandsrecht, internationales Privat- und Prozeßrecht an der Universität Hamburg, die „Forschungsstelle für Kriegsursachen“, später „Archiv der Friedensverträge", die die deutsche Dokumentensammlung zur Vorgeschichte des Ersten Weltkriegs unter dem Titel „Große Politik der Europäischen Kabinette 1871-1914“ herausgab. Im Jahr 1923 wurde mit Unterstützung des Hamburgischen Staates die „Forschungstelle“ zu der privatrechtlichen Stiftung „Institut für Auswärtige Politik " erweitert. Dem Institut gehörten Historiker, Juristen, Volkswirtschaftler und Journalisten an, um bei der Erforschung der politischen Verhältnisse alle denkbaren Aspekte gebührend berücksichtigen zu können. Ihm standen eine rasch wachsende Bibliothek zur Auswärtigen Politik und ein umfangreiches zeitgeschichtliches Archiv in Gestalt einer Zeitungsausschnittsammlung zur Verfügung. An die Offentlichkeit wandte es sich regelmäßig mit seiner Zeitschrift „Europäische Gespräche“. In dieser Zeitschrift erschien beispielsweise im Jahre 1926 eine auch heute noch sehr interessante Darstellung des kolonialpolitischen Interesses der deutschen Elite ${ }^{3}$.

Das Jahr 1933 bezeichnet den folgenschwersten Einschnitt in der Entwicklung des Instituts. Mendelssohn-Bartholdy emigrierte 1934 nach Großbritannien, die Zeit-

2 Friedrich Schack, Das deutsche Kolonialrecht bis zum Weltkriege, Hamburg 1923, S. XII und S. 392.

3 Eine Umfrage: Soll Deutschland Kolonialpolitik treiben?, 1926, S. 609 ff. 
schrift wurde eingestellt, und trotz aller Vorkehrungen der Universität wurde das Institut im Jahre 1937 nach Berlin verlegt und dem „Deutschen Institut für Außenpolitische Forschung" angeschlossen. Bibliothek und Archiv blieben allerdings auch dort als selbständige Einheit erhalten und wurden weiter ausgebaut. Im Jahre 1943 wurde das Institut einschließlich des Personals aus Berlin nach Thüringen verlegt. Dort gelangte es 1945 in die Hände amerikanischer Truppen. Im Jahre 1946 gelang es, von der amerikanischen Militärregierung die Rückgabe an die Stadt Hamburg zu erreichen. Bereits im Frühjahr 1947 wurde das Material des Instituts, das nach den kriegsbedingten Verlusten noch eine Bibliothek von ca. 30000 Bänden und das vollständige Archiv von außenpolitisch bedeutsamen Presseausschnitten und Rundfunkmeldungen der Jahre 1911-1945 umfaßte, der Öffentlichkeit wieder zugänglich gemacht. Zugleich wurde die Geschäftsführung des Instituts der neugegründeten Forschungsstelle für Völkerrecht und ausländisches öffentliches Recht der Universität Hamburg übertragen, die 1957 das Institut mit ihrem eigenen Forschungsstab in einem eigenen Gebäude vereinigen konnte (Hamburg, Mittelweg 186).

Das Institut für Auswärtige Politik ist heute im wesentlichen Bibliothek und zeitgeschichtliches Archiv. Als solches ist es mit einem Bestand von zur Zeit ca. 60000 Bänden (darunter ca. 3000 Zeitschriftentitel) und ca. 1,2 Millionen Zeitungsausschnitten die größte öffentlich zugängliche Sammlung zur Auswärtigen Politik in Deutschland4. Die Schwerpunkte der Sammlung sind: Außenpolitik der europäischen Staaten seit 1871, Theorie der Auswärtigen Politik, Außen- und Innenpolitik der nicht-europäischen Staaten ${ }^{5}$. Für das Archiv werden laufend führende Tageszeitungen aus Afrika, Amerika, Asien und Europa ausgewertet ${ }^{6}$. Die gesamten Kosten des Instituts trägt heute die Freie und Hansestadt Hamburg.

\section{Die Forschungsstelle für Völkerrecht und ausländisches öffentliches Recht der Universität Hamburg}

Die Forschungsstelle ist ein Hamburgisches Staatsinstitut an der Universität. Sie dient ausschließlich der Forschung. Ihre Forschungsaufgaben erstrecken sich auf das Gebiet des Völkerrechts, der Verfassungsentwicklung im weitesten Sinne und die internationalen Beziehungen. Direktor der Forschungsstelle ist seit 1955 Professor Herbert Krüger, zugleich Ordinarius für öffentliches Recht, Völkerrecht und Auswärtige Politik an der Universität Hamburg. Das Institut ist in folgende Referate gegliedert:

1. Ausländisches Verfassungsrecht und Staatsangehörigkeitsrecht (1 Referent)

2. Internationales öffentliches Seerecht ( 3 Referenten, davon einer zugleich mit der Geschäftsführung der Forschungsstelle beauftragt)

3. Verfassungsentwicklung und internationale Beziehungen der sozialistischen Staaten (1 Referent)

4. Verfassungsentwicklung und internationale Beziehungen der neuen Staaten in Übersee (1 Referent)

5. Internationale Beziehungen der atlantischen Staaten (1 Referent beim Institut für Auswärtige Politik, zugleich bibliographischer Berater dieses Instituts)

Das ständige wissenschaftliche Personal wird ergänzt durch Gastreferenten (1969 zwei) und durchschnittlich zehn externe Mitarbeiter mit Forschungsaufträgen (vorwiegend Doktoranden). Das gesamte ständige Personal der Forschungsstelle und des

4 Die größte Sammlung dieses Bereiches besitzt das Auswärtige Amt in Bonn; sie ist allerdings nur beschränkt zugänglich.

5 Die Literatur zur Innenpolitik der lateinamerikanischen Staaten wird vorwiegend bei der Staats- und Universitätsbibliothek Hamburg gesammelt, die Literatur in nicht-lateinischen Schriften bei den Hamburger Regionalinstituten.

6 X Whnliche Archive mit vertieften Sammlungen für die regionale Politik bestehen bei den vier Hamburger Länderinstituten. 
mit ihr heute fest verbundenen Instituts für Auswärtige Politik zählt im Jahre 1969 zwanzig Personen. Den größten Teil der Personallasten trägt die Freie und Hansestadt Hamburg.

Es ist unmöglich, im Rahmen dieses Berichtes die bald 200 Veröffentlichungen der Forschungsstelle $\mathrm{zu}$ erwähnen, geschweige $\mathrm{zu}$ würdigen (das Gesamtverzeichnis „Sämtliche Veröffentlichungen 1948-1965“ mit Ergänzung wird Interessenten kostenlos zur Verfügung gestellt). Es können nur kurz die elf Reihen mit ihren thematischen Schwerpunkten und ihren für die Thematik dieser Zeitschrift wichtigsten Titeln genannt werden.

a) "Abhandlungen“ (Alfred Metzner Verlag, Frankfurt am Main und Berlin), grundlegende Untersuchungen vor allem zum Völkerrecht ${ }^{7}$.

b) „Darstellungen zur Auswärtigen Politik“, herausgegeben von Professor Dr. Herbert Krüger (veranstaltet von der Hamburger Gesellschaft für Völkerrecht und auswärtige Politik), historische und politische Studien zu Problemen der Staaten Asiens, Afrikas und Lateinamerikas 8 .

c) „Dokumente" (herausgegeben in Verbindung mit den völkerrechtlichen Instituten in Göttingen und Kiel, Verlag Alfred Metzner, Frankfurt am Main und Berlin), thematisch geordnete Dokumentensammlungen zum Völkerrecht und zum Verfassungsrecht ${ }^{9}$.

d) "Das geltende Seekriegsrecht in Einzeldarstellungen“, herausgegeben von Professor Dr. Herbert Krüger und Professor Dr. Rolf Stödter, Untersuchungen zu den wichtigen Instituten des Seekriegsrechts unter besonderer Berücksichtigung von Belangen der neutralen Handelsschiffahrt.

e) "Hamburger öffentlich-rechtliche Nebenstunden“, herausgegeben von Professor Dr. Herbert Krüger, vorwiegend Gelegenheitsarbeiten von Angehörigen der Forschungsstelle zur allgemeinen Staats- und Rechtslehre.

f) "Sammlung geltender Staatsangehörigkeitsgesetze“ (Alfred Metzner Verlag, Frankfurt am Main und Berlin), einführende Kommentare und Sammlung aller jemals gültigen Staatsangehörigkeitsbestimmungen eines Staates mit einem Register der Literatur und neuerdings auch der Rechtsprechung. Bisher liegen die Bände für 76 Staaten vor ${ }^{10}$.

7 Bd. 9: Moosmayer, Peter: Der Gebietsgrundsatz im Staatsangehörigkeitsrecht (ius soli), unter besonderer Berücksichtigung der südamerikanischen Staaten, 1963.

8 Bd. 1: Gehrke, Ulrich: Persien in der deutschen Orientpolitik während des Ersten Weltkrieges, Hamburg 1960;

Bd. 2: Gehrke, Ulrich - Kuhn, Gustav: Die Grenzen des Irak - Historische und rechtliche Aspekte des irakischen Anspruchs auf Kuwait und des irakisch-persischen Streites um den Schatt al-Arab, Hamburg 1963;

Bd. 3: Soerjono Wirjodiatmodjo, Raden: Der Gedanke der Blockfreiheit in Südostasien - Geschichte und Deutung der Solidaritätskonferenzen der Colombo-Staaten 1954-1961, Hamburg 1964;

Bd. 4: Quaison-Sackey, Alex: Afrika ohne Fesseln - Gedanken eines afrikanischen Politikers (Ubersetzung aus dem Englischen), Hamburg 1965;

Bd. 5: Doll, Peter: Der senegalesische Weg zum afrikanischen Sozialismus - Konzeption und Verwirklichung der senegalesischen Entwicklungspolitik, Hamburg 1966;

Bd. 6: Lang, Gerhard: Boliviens Streben nach freiem Zugang zum Meer - Ein Beitrag zur Geschichte des neueren Völkerrechts und der Diplomatie, Hamburg 1966;

Bd. 7: Schröder, Dieter: Die Konferenzen der "Dritten Welt“ - Solidarität und Kommunikation zwischen nachkolonialen Staaten, Hamburg 1968.

9 Bd. VIII: Wicharz, Michael: Die Ernährungs- und Landwirtschaftsorganisation der Vereinten Nationen (FAO), Satzung und Erklärung, Beschlüsse und Empfehlungen der Ernährungs- und Landwirtschafts-

konferenz der Vereinten Nationen (Hot Springs), 1953;
Bd. XXIV A: Hecker, Hellmuth: Verfassungsregister, Teil III: Amerika, 1958;

Bd. XXIV B: Hecker, Hellmuth, Verfassungsregister, Teil IV: Afrika - Asien - Australien, Nachweise

von Dokumenten zur politischen und staatsrechtlichen Geschichte der Entwicklungsländer, 1963;

Bd. XXVII: Sasse, Horst: Die asiatisch-afrikanischen Staaten auf der Bandung-Konferenz, 1958;

Bd. XXXVI: Hoog, Günter: Die Genfer Seerechtskonferenzen von 1958 und 1960, 1961;

Bd. XXXVII: Tomson, Edgar: Die Volksrepublik China und das Recht nationaler Minderheiten, 1963.

10 Bd. 13: Israel und das ehemalige Mandatsgebiet Palästina, 1954;

Bd. 14: Südafrika einschließlich Südwest-Afrika, 1955;

Bd. 15: Arabische Staaten, 1954;

Bd. 19: Brasilien und Chile, 1957;

Bd. 23: Kolumbien, Ekuador und Venezuela, 1960;

Bd. 24: Bolivien und Perú, 1963;

Bd. 26: Indien, Pakistan und Nepal, 1965;

Bd. 27: Italien einschließlich der ehemaligen Kolonien, 1968;

Bd. 28: Französisch-sprachiges Schwarz-Afrika: Dahome, Elfenbeinküste, Gabun, Guinea, Kamerun, Kongo, Madagaskar, Mali, Mauretanien, Niger, Obervolta, Senegal, Togo, Tschad, Zentralafrikanische Republik, 1968. 
g) „Schriften zur Auswärtigen Politik“, herausgegeben von Professor Dr. Herbert Krüger, historische Arbeiten ${ }^{11}$.

h) „Die Staatsverfassungen der Welt in Einzelausgaben“, kommentierte Sammlung aller jemals gültigen Verfassungstexte der modernen Staaten; bisher sind neun Staaten bearbeitet ${ }^{12}$.

i) „Untersuchungen zur Auswärtigen Politik“, herausgegeben von Professor Dr. Herbert Krüger (Alfred Metzner Verlag, Frankfurt am Main und Berlin), grundlegende Analysen zu Fragen der Auswärtigen Politik und des Völkerrechts ${ }^{13}$.

k) „Varia iuris publici“ (früher „Hektographierte Veröffentlichungen“), Arbeiten aus der Gutachtertätigkeit des Instituts und qualifizierte Dissertationen zum Völkerrecht und zum öffentlichen Recht.

1) „Werkhefte“, Zusammenstellungen von Materialien und kurze Stellungnahmen $\mathrm{zu}$ aktuellen völkerrechtlichen Themen ${ }^{14}$.

Nach diesem Úberblick über einen kleinen Teil des Publikationsprogramms der Forschungsstelle wäre es müßig, noch lange Darlegungen über die Methoden und Arbeitsgebiete zu geben, zumal schon im ersten Heft dieser Zeitschrift mit dem Programmaufsatz das zukünftige Programm des Instituts im Bereich der Úberseeforschung umrissen worden ist.

D.S.

11 Bd. 1: Dunker, Hans Joachim: Die interamerikanischen Beziehungen und der Gedanke der gleichberechtigten Partnerschaft, 1957;

Bd. 3: Plass, Jens, England zwischen Rußland und Deutschland - Der Persische Golf in der britischen Vorkriegspolitik 1899-1907, 1966;

Bd. 5: Peters, Klaus: Die Herrschaftsordnung von Buganda - ein Weg zu institutionalisierter Herrschaft im alten Afrika, 1968.

12 Bd. 2: Ansari, Adnan: Die Verfassungen des Libanon, der Vereinigten Arabischen Republik und des Irak, 1960;

Bd. 4: Röhl, Wilhelm: Die japanische Verfassung, 1963;

Bd. 5: Wenk, Klaus: Die Verfassungen Thailands, 1964;

Bd. 6: Prieur Koelling, Wolfgang: Die Verfassung von Chile, 1964.

13 Bd. 1: Oppermann, Thomas: Die Algerische Frage, 1959;

Bd. 3: Ahrens, Dieter: Der Karibische Raum als Interessensphäre der Vereinigten Staaten, 1965;

Bd. 4; Krakau, Knud: Die kubanische Revolution und die Monroe-Doktrin, 1968.

$14 \mathrm{Heft}$ 9: Kehden, Max Ivers - Henkmann, Marie-Louise: Die Inanspruchnahme von Meereszonen durch Küstenstaaten - Eine Ubersicht über die Staatenpraxis, 1967;

Heft 10: Schröder, Dieter: Der freie Zugang der Binnenstaaten zum Meer - Die internationalen Ubereinkommen, 1966. 